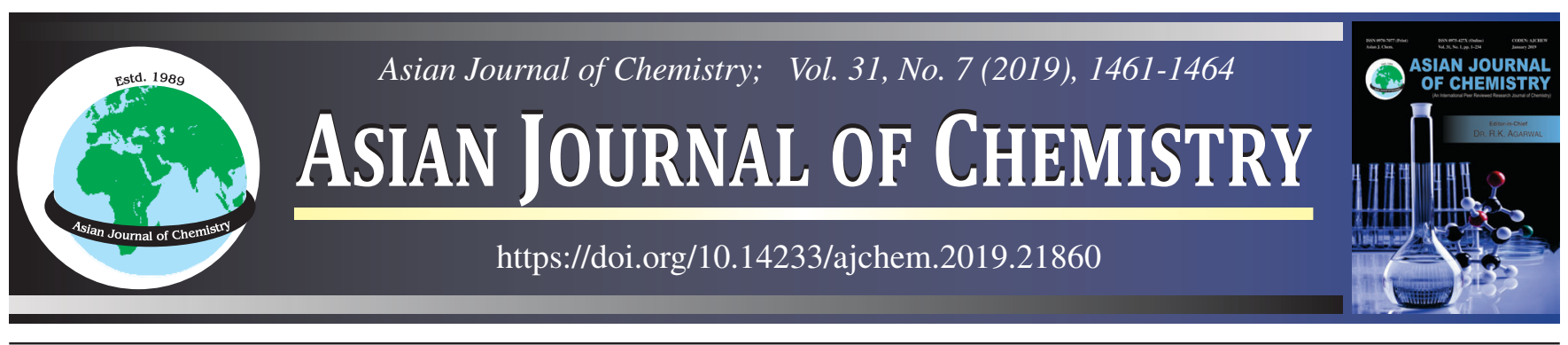

\title{
Synthesis of Substituted 4-(4-((3-Nitro-2-oxo-2H-chromene-4- yl)amino)phenyl)morpholine-3-one Coumarin Derivatives
}

\author{
Yogesh J. Sanghani ${ }^{1, *}$, , SuResh B. Koradiya ${ }^{2}$ and Anilkumar S. Patel ${ }^{2}$
}

${ }^{1}$ Department of Chemistry, School of Science, R K University, Rajkot-360020, India

${ }^{2}$ Department of Chemistry, Shree M. \& N. Virani Science College, Rajkot-360005, India

*Corresponding author: E-mail: yogeshsanghani481@gmail.com

Received: 30 November 2018;

Accepted: 24 January 2019;

Published online: 21 May 2019;

AJC-19396

\begin{abstract}
A series of novel 4-(4-amino phenyl) morpholine-3-one substituted coumarin derivatives have been prepared by chloramine coupling reaction and were identified. The novel synthetic route involves nucleophilic substitution reaction of 4-chloro-3-nitro- $2 \mathrm{H}$-chromene-2one with 4-(4-amino phenyl)morpholine-3-one. Due to the presence of nitro group in coumarin derivatives make substitution reaction easy and convenient at low temperature. Using DMF as solvent and $\mathrm{K}_{2} \mathrm{CO}_{3}$ as base various substituted 4-(4-((3-nitro-2-oxo-2H-chromen4-yl)amino)phenyl)morpholine-3-one derivatives (YS-1 to YS-10) can be obtain in good yield and high purity. Structural characterization of all synthesized compound was done by NMR, Mass and IR spectra.
\end{abstract}

Keywords: 4-Chloro-3-nitro-2H-chromene-2-one, 4-(4-Amino phenyl)morpholine-3-one, 4-Hydroxy coumarin.

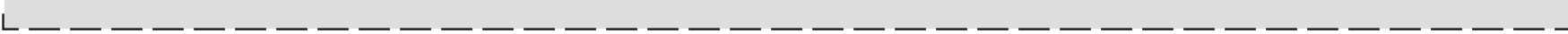

\section{INTRODUCTION}

In the large family of heterocyclic chemistry, coumarin and its derivatives have significant role due to their distinct applications, abundant availability in nature and various routes for its synthesis. The main aim of this research is to synthesize 4-(4-amino phenyl)morpholine-3-one substituted coumarin derivatives. Some researchers $[1,2]$ reported several natural and synthetic coumarin derivatives which behaves as antimicrobial agents. Novobiocin and chlorobiocin are important class of antimicrobials compounds containing coumarin ring [3].

Some substituted coumarin derivatives have antibacterial potential and DNA Gyrase inhibitor potential [4]. Di Braccio et al. [5] synthesized coumarin based hydrazine derivative which have antimicrobial property. In the present work, we report the synthesis of 4-(4-((3-nitro-2-oxo-2H-chromen-4-yl)amino)phenyl)morpholine-3-one by a novel synthetic route by chloroamine coupling. The interesting chemical and physical properties and the pharmacological effects of aminocoumarin derivatives were the main motivation for starting this research [6]. At first, the 4-amino compound was synthesized as a starting compound for the synthesis of large spectra of new coumarin derivatives [7].
We have designed a series of compounds incorporating coumarin and morpholine moeities in the structure in compounds have been synthesized via simple steps. A series of novel 4-(4-amino phenyl)morpholine-3-one substituted coumarin derivatives have been prepared by chloramine coupling [8-10] reaction and were identified by common spectroscopic methods.

\section{EXPERIMENTAL}

All the chemicals and solvents used in the reaction were used of Rankem Pvt. Ltd. of analytical grade and hence no need to further purification. Melting points of synthesized compounds was taken by open capillary method. FTIR-8400 spectrophotometer (Shimadzu, Kyoto, Japan) is used for IR spectra characterization, using DRS probe $\mathrm{KBr}$ pallet. The Brucker-Avance II (400 MHz) instrument were used for NMR analysis and $\mathrm{CDCl}_{3}$ used as a solvent. For mass analysis we used GCMS-QP-2010 spectrometer.

Synthesis of INT-a: To well stir round bottom flask containing aniline $(0.01 \mathrm{~mol})$, ethylene oxide gas passed [11]. Reaction was continuously monitored on thin layer chromatography. At the end, reaction mixture was cooled and quince in ice water to get INT-a (Scheme-I).

This is an open access journal, and articles are distributed under the terms of the Creative Commons Attribution-NonCommercial-ShareAlike 4.0 (CC BY-NC-SA 4.0) International License which allows readers to freely read, download, copy, distribute, print, search, or link to the full texts of its articles and to use them for any other lawful non-commercial purpose as long as the original source is duly acknowledged. 
Synthesis of INT-b: Chloroacetyl chloride was added in drop wise manner in a previously cooled round bottom flask containing INT-a $(0.01 \mathrm{~mol}), \mathrm{DMF}$ and $\mathrm{K}_{2} \mathrm{CO}_{3}(0.02 \mathrm{~mol})$ maintain the temperature at $0{ }^{\circ} \mathrm{C}$. After completion of the addition, raise the temperature at $60^{\circ} \mathrm{C}$ and maintain further $4 \mathrm{~h}$ progress of the reaction was monitored on TLC (Scheme-I).

Synthesis of INT-c: In a $250 \mathrm{~mL}$ round bottom flask, $\mathrm{H}_{2} \mathrm{SO}_{4}(0.03 \mathrm{~mol})$ was taken and cooled to $0{ }^{\circ} \mathrm{C}$. To this $\mathrm{HNO}_{3}$ $(0.03 \mathrm{~mol})$ was added in a drop-wise manner [12]. To this nitrating mixture INT-b (0.01 mole) was added portion wise. After completion of the addition, reaction mixture was stirred at $60^{\circ} \mathrm{C}$ for $3 \mathrm{~h}$ reaction mixture was cooled and quince in ice water, after the confirm on TLC that reaction was completed (Scheme-I).

Synthesis of INT-d: In a $250 \mathrm{~mL}$ round bottom flask, hydrochloric acid $(3 \mathrm{~V})$ was taken and cools to $5{ }^{\circ} \mathrm{C}$. To this tin metal was added. In this reaction mixture, INT-b $(0.01 \mathrm{~mol})$ was added and heat the reaction mixture at $70{ }^{\circ} \mathrm{C}$ for $5 \mathrm{~h}$. Reaction mixture was cooled and quince in water after the completion of the reaction [13]. Neutralize the mixture with $\mathrm{NaOH}$ solution until neutral $\mathrm{pH}$. Extract the reaction mass with ethyl acetate and evaporate ethyl acetate to give INT-d (Scheme-I).

Synthesis of 4-hydroxy coumarin (INT-1): Take malonic acid $(0.1 \mathrm{~mol})$ and substituted phenol $(0.1 \mathrm{~mol})$ in a $250 \mathrm{~mL}$ round bottom flask. To this add phosphorous oxy chloride (40 $\mathrm{mL}$ ) and previously dried anhydrous zinc chloride ( $30 \mathrm{~g})$. After the addition raise the temperature at $70{ }^{\circ} \mathrm{C}$ and maintain this temperature for 8-10 h in a water bath [14]. Reaction mixture was cooled and quince in crushed ice to afford INT-1 as a solid. Filter the solid and wash with water until free from acid. The separated solid product treated with $10 \%$ sodium bicarbonate solution and filter again to remove undisclosed residue [15].
Finally the filtrate was cooled and acidifies with dilute hydrochloric acid. Cool and settle down the particle and filter again to get pure INT-1 (Scheme-I).

General synthesis of various substituted 4-hydroxy 3nitro coumarin (INT-2): Nitration of various substituted 4-hydroxy coumarin was carried out with $\mathrm{HNO}_{3}$ and acetic acid at $80-85^{\circ} \mathrm{C}$ for $1.5 \mathrm{~h}$ to afford-nitro substituted coumarin. In a round bottom flask, nitric acid ( 2 equivalent) and acetic acid $(5 \mathrm{~mL})$ was mixed and substituted 4-hydroxy coumarin was added. After the addition raise the temperature at $80-85^{\circ} \mathrm{C}$ temperature for $1.5 \mathrm{~h}$ reaction mixture was cooled and poured into crushed ice to afford yellow coloured solid of 3-nitro 4hydroxy. Synthesized 3-nitro 4-hydroxy coumarin was characterized by mass, NMR and elemental analysis (Scheme-I).

General synthesis of various substituted 4-chloro 3nitro coumarin (INT-3): In a round bottom flask, $\mathrm{POCl}_{3}(2.5$ equivalent) was cooled at $0{ }^{\circ} \mathrm{C}$ and to this DMF was added drop wise for $20 \mathrm{~min}$ and maintain the temperature at $0{ }^{\circ} \mathrm{C}$ then added 4-hydroxy 3-nitro coumarin portion wise to cool the solution [16]. After the addition leave the reaction mixture stir further for $1 \mathrm{~h}$ at the end, cool the reaction mixture and poured into the crushed ice and filter the separated product to afford yellowish 4-chloro 3-nitrocoumarin (Scheme-I) [17,18].

General synthesis 4-(4-((3-nitro-2-oxo-2H-chromen-4yl)amino)phenyl)morpholine-3-one derivatives: In a round bottom flask, $10 \mathrm{~mL}$ IPA was cooled and stirred at $0-5{ }^{\circ} \mathrm{C}$, to this substituted 4-chloro-3-nitro- $2 \mathrm{H}$-chromen-2-one $(0.01 \mathrm{~mol})$ was added followed by addition of 4-(4-aminophenyl)morpholine-3-one $(0.1 \mathrm{~mol})$ and potassium carbonate $(0.01 \mathrm{~mol})$ was added to the reaction mass [19]. After the addition over, leave the reaction mixture stir at room temperature for $30 \mathrm{~min}$ maintain the temperature below $10{ }^{\circ} \mathrm{C}$, after the completion of the reaction evaporate the solvent under vacuum and wash the<smiles>[R]c1cccc(O)c1</smiles>

Scheme-I 
residue with cooled ice water to gives 4-(4-((3-nitro-2-oxo$2 \mathrm{H}$-chromen-4-yl)amino)phenyl)morpholine-3-one in good yield (Scheme-I). Purification was done by treating solid mass with dilute hydrochloric acid [20].

\section{Spectral data}

4-(4-((3-Nitro-2-oxo-2H-chromen-4-yl)amino)phenyl)morpholine-3-one (YS-01): Yield: $70 \%$; m.p.: 216-218 ${ }^{\circ} \mathrm{C}$; m.f.: $\mathrm{C}_{19} \mathrm{H}_{15} \mathrm{~N}_{3} \mathrm{O}_{6}$, m.w.: 381.34 , Appearance: Colourless white, $\mathrm{R}_{\mathrm{f}}$ value-0.41 (8:2 ethyl acetate:hexane), ${ }^{1} \mathrm{H}$ NMR spectra in $\delta$ ppm: 3.80 to $3.88 \delta$ ppm (triplet, $2 \mathrm{H},-\mathrm{CH}_{2}-\mathrm{O}-$ ), 4.03 to $4.11 \delta$ ppm (triplet, $2 \mathrm{H},-\mathrm{N}-\mathrm{CH}_{2}-$ ), $4.37 \delta \mathrm{ppm}$ (singlet, $2 \mathrm{H}$, $-\mathrm{CO}-\mathrm{CH}_{2}-$ ), 7.0 to $7.6 \delta \mathrm{ppm}$ ( $8 \mathrm{H}$ aromatic region), $11.2 \delta \mathrm{ppm}$ (singlet, $1 \mathrm{H},-\mathrm{NH}-)$, IR $\left(\mathrm{KBr}, v_{\max }, \mathrm{cm}^{-1}\right): 3279,3076,2864,1712,1650$, $1612,1551,1513,1452,1350,1307,1211,1120,1061,996$, $853,753,692,626$. Mass spectra $m / z: 381\left(\mathrm{M}^{+}\right), 363,336$, $318,290,277,262,248,235,221,207,{ }^{13} \mathrm{C}$ NMR $(100 \mathrm{MHz}$, chloroform- $d$ ) $\delta: 132.17,125.77,123.30,122.93,121.53,116.19$. Elemental analysis (\%): C, 59.84; H, 3.96; N, 11.02; Found (\%): C, 59.12; H, 3.92; N, 11.09 .

4-(4-((8-Chloro-3-nitro-2-oxo-2H-chromen-4-yl)amino)phenyl)morpholine-3-one (YS-02): Yield: $60 \%$; m.p.: 202-204 ${ }^{\circ} \mathrm{C}$; m.f.: $\mathrm{C}_{19} \mathrm{H}_{14} \mathrm{~N}_{3} \mathrm{O}_{6} \mathrm{Cl}$, m.w.: 415.78, Appearance: Colourless white, $\mathrm{R}_{\mathrm{f}}$ value- 0.42 (8:2 ethyl acetate:hexane), ${ }^{1} \mathrm{H}$ NMR spectra in $\delta$ ppm: 3.79 to $3.89 \delta \mathrm{ppm}$ (triplet, $2 \mathrm{H},-\mathrm{CH}_{2^{-}}$ $\mathrm{O}-$ ), 4.01 to $4.11 \delta \mathrm{ppm}$ (triplet, $2 \mathrm{H},-\mathrm{N}_{-} \mathrm{CH}_{2}-$ ), $4.38 \delta \mathrm{ppm}$ (singlet, $2 \mathrm{H}$, - $\mathrm{CO}-\mathrm{CH}_{2}-$ ), 6.9 to $7.4 \delta \mathrm{ppm}$ (7H aromatic region), $11.1 \delta \mathrm{ppm}$ (singlet, $1 \mathrm{H},-\mathrm{NH}-)$. IR $\left(\mathrm{KBr}, \mathrm{v}_{\max }, \mathrm{cm}^{-1}\right)$ : 3281 , 3077, 2865, 1710, 1651, 1615, 1561, 1514, 1438, 1351, 1307, $1212,1121,1059,998,851,752,692,625$. Mass spectra $\mathrm{m} / \mathrm{z}$ : $415\left(\mathrm{M}^{+}\right)(100.0 \%), 417.05(32.0 \%), 416.06(20.9 \%), 418.06$ (7.0\%), $417.06(3.5 \%), 416.05(1.1 \%), 419.06(1.1 \%), 363$, $336,318,290,277,262,248,235,221,207,{ }^{13} \mathrm{C}$ NMR (100 MHz, chloroform- $d$ ) $\delta: 133.07,124.83,123.91,123.30,121.53$. Elemental analysis (\%): C, 54.89; H, 3.39; Cl, 8.53; N, 10.11; Found (\%): C, 49.27; H, 3.40; Cl, 8.50; N, 9.11.

4-(4-((6-Chloro-3-nitro-2-oxo-2H-chromen-4-yl)amino)phenyl)morpholine-3-one (YS-03): Yield: 65 \%; m.p.: 296$298^{\circ} \mathrm{C}$; m.f.: $\mathrm{C}_{19} \mathrm{H}_{14} \mathrm{~N}_{3} \mathrm{O}_{6} \mathrm{Cl}$, m.w.: 415.78, Appearance: Colourless white, $\mathrm{R}_{\mathrm{f}}$ value- 0.43 (8:2 ethyl acetate:hexane), ${ }^{1} \mathrm{H}$ NMR spectra in $\delta$ ppm: 3.80 to $3.89 \delta$ ppm (triplet, $2 \mathrm{H},-\mathrm{CH}_{2}-\mathrm{O}-$ ), 4.02 to $4.16 \delta \mathrm{ppm}$ (triplet, $2 \mathrm{H},-\mathrm{N}-\mathrm{CH}_{2}-$ ), $4.32 \delta \mathrm{ppm}$ (singlet, $\left.2 \mathrm{H},-\mathrm{CO}-\mathrm{CH}_{2}-\right), 6.8$ to $7.4 \delta \mathrm{ppm}(7 \mathrm{H}$ aromatic region), $11.3 \delta$ ppm (singlet, $1 \mathrm{H},-\mathrm{NH}-)$. IR $\left(\mathrm{KBr}, v_{\max }, \mathrm{cm}^{-1}\right): 3280,3077,2864$, 1711, 1650, 1616, 1541, 1539, 1442, 1352, 1307, 1212, 1131, $1049,997,851,752,690,625$. Mass spectra $m / z: 415.06\left(\mathrm{M}^{+}\right)$ (100.0\%), $417.05(32.0 \%), 416.06$ (20.9\%), $418.06(7.0 \%)$, 417.06 (3.5\%), $416.05(1.1 \%), 419.06(1.1 \%), 363,336$, $318,290,277,262,248,235,221,207,{ }^{13} \mathrm{C}$ NMR $(100 \mathrm{MHz}$, chloroform- $d$ ) $\delta:$ 132.85, 125.44, 123.30, 121.53, 117.56. Elemental analysis (\%): C, 54.89; H, 3.39; Cl, 8.53; N, 10.11; Found (\%): C, 49.27; H, 3.40; Cl, 8.50; N, 9.11.

4-(4-((6-Fluoro-3-nitro-2-oxo-2H-chromen-4-yl)amino)phenyl)morpholine-3-one (YS-04): Yield: $74 \%$; m.p.: 234-236 ${ }^{\circ} \mathrm{C}$; m.f.: $\mathrm{C}_{19} \mathrm{H}_{14} \mathrm{~N}_{3} \mathrm{O}_{6} \mathrm{~F}$, m.w.: 399.33, Appearance: Colourless white, $\mathrm{R}_{\mathrm{f}}$ value- 0.42 (8:2 ethyl acetate:hexane), ${ }^{1} \mathrm{H}$ NMR spectra in $\delta \mathrm{ppm}: 3.80$ to $3.91 \delta \mathrm{ppm}$ (triplet, $2 \mathrm{H},-\mathrm{CH}_{2^{-}}$ $\mathrm{O}-$ ), 4.02 to $4.16 \delta \mathrm{ppm}$ (triplet, $2 \mathrm{H},-\mathrm{N}_{-} \mathrm{CH}_{2}-$ ), $4.30 \delta \mathrm{ppm}$ (singlet, $2 \mathrm{H},-\mathrm{CO}-\mathrm{CH}_{2}-$ ), 6.9 to $7.6 \delta \mathrm{ppm}$ ( $7 \mathrm{H}$ aromatic region), $11.2 \delta \mathrm{ppm}$ (singlet, $1 \mathrm{H},-\mathrm{NH}-)$, IR ( $\left.\mathrm{KBr}, v_{\max }, \mathrm{cm}^{-1}\right): 3271$, $3258,2866,2282$, 1692, 1654, 1545, 1538, 1439, 1315, 1309, $1218,1139,1040,878,851,768,655$. Mass spectra $\mathrm{m} / z: 399.09$ (100.0\%), 400.09 (20.9\%), 401.09 (3.5\%), 400.08 (1.1\%), 363. 336. 318. 290. 277. 262. 248. 235. 221. 207, ${ }^{13} \mathrm{C}$ NMR (100 MHz, chloroform- $d$ ) $\delta: 123.30,121.53,120.14,119.94$, 117.45, 117.37, 112.42, 112.22, Elemental analysis (\%): C, 57.15; H, 3.53; F, 4.76; N, 10.52; Found (\%): C, 57.10; H, $3.50 ; \mathrm{F}, 4.70 ; \mathrm{N}, 10.20$.

4-(4-((6-Bromo-3-nitro-2-oxo-2H-chromen-4-yl)amino)phenyl)morpholine-3-one (YS-05): Yield: $78 \%$; m.p.: 244-246 ${ }^{\circ} \mathrm{C}$; m.f.: $\mathrm{C}_{19} \mathrm{H}_{14} \mathrm{~N}_{3} \mathrm{O}_{6} \mathrm{Br}$, m.w.: 460.23 , Appearance: Colourless white, $\mathrm{R}_{\mathrm{f}}$ value-0.40 (8:2 ethyl acetate:hexane), ${ }^{1} \mathrm{H}$ NMR spectra in $\delta$ ppm: 3.80 to $3.92 \delta$ ppm (triplet, $2 \mathrm{H}$, $-\mathrm{CH}_{2-}$ $\mathrm{O}-$ ), 4.03 to $4.17 \delta \mathrm{ppm}$ (triplet, $2 \mathrm{H},-\mathrm{N}_{-} \mathrm{CH}_{2^{-}}$), $4.31 \delta \mathrm{ppm}$ (singlet, $2 \mathrm{H},-\mathrm{CO}-\mathrm{CH}_{2}-$ ), 6.7 to $7.4 \delta \mathrm{ppm}$ (7H aromatic region), $11.2 \delta \mathrm{ppm}$ (singlet, $1 \mathrm{H},-\mathrm{NH}-$ ). IR ( $\left.\mathrm{KBr}, v_{\max }, \mathrm{cm}^{-1}\right)$ : 3371 , $3358,2966,2282,1692,1654,1546,1510,1439,1325,1310$, $1218,1129,1062,870,851,761,655$. Mass spectra $m / z: 459.01$ (100.0\%), 461.00 (97.3\%), 460.01 (20.9\%), 462.01 (20.7 $\%), 461.01$ (3.5\%), 463.01 (3.2\%), 460.00 (1.1\%), 462.00 (1.1\%), 363, 336, 318, 290, 277, 262, 248, 235, 221, 207, ${ }^{13} \mathrm{C}$ NMR (100 MHz, chloroform-d) $\delta: 135.16,128.23,123.30$, 121.53, 117.49. Elemental analysis (\%): C, 49.58; H, 3.07; Br, 17.36; N, 9.13; Found (\%): C, 49.34; H, 3.01; Br, 17.10; N, 8.18.

4-(4-((6,8-Dimethyl-3-nitro-2-oxo-2H-chromen-4-yl)amino)phenyl)morpholine-3-one (YS-06): Yield: $70 \%$; m.p.: 216-218 ${ }^{\circ} \mathrm{C}$; m.f.: $\mathrm{C}_{21} \mathrm{H}_{19} \mathrm{~N}_{3} \mathrm{O}_{6}$, m.w.: 409.13, Appearance: Off white, $\mathrm{R}_{\mathrm{f}}$ value- 0.40 (8:2 ethyl acetate:hexane), ${ }^{1} \mathrm{H}$ NMR spectra in $\delta \mathrm{ppm}: 2.41 \delta \mathrm{ppm}$ (singlet, $6 \mathrm{H},-\mathrm{CH}_{3}$ ), 3.80 to $3.89 \delta \mathrm{ppm}$ (triplet, $2 \mathrm{H},-\mathrm{N}-\mathrm{CH}_{2}$ ), 4.11 to $4.18 \delta \mathrm{ppm}$ (triplet, $2 \mathrm{H}$, -CO$\left.\mathrm{CH}_{2}-\right), 6.89$ to $7.88 \delta \mathrm{ppm}(6 \mathrm{H}$, aromatic region), $11.02 \delta \mathrm{ppm}$ (singlet, $1 \mathrm{H},-\mathrm{NH})$. IR $\left(\mathrm{KBr}, \mathrm{v}_{\max }, \mathrm{cm}^{-1}\right): 3779,3734,3663$, 3643, 3391, 3268, 2920, 2371, 2354, 2281, 1691, 1656, 1597, 1557, 1521, 1486, 1416, 1367, 1311, 1238, 1193, 1125, 1068, $999,875,820,766,752,655$. Mass spectra $m / z: 409.13(100.00$ $\%), 410.13(23.2 \%), 411.13$ (4.0\%), 410.12 (1.1\%), 395, $378,365,361,349,332,306,289,275,262,249,223,278$, $205,{ }^{13} \mathrm{C}$ NMR (100 MHz, chloroform- $d$ ) $\delta: 135.60,123.30$, 121.53, 121.29, 20.69, 15.95. Elemental analysis (\%): C, 61.61; H, 4.68; N, 10.26; Found (\%): C, 60.02; H, 4.37; N, 9.53.

4-(4-((7,8-Dimethyl-3-nitro-2-oxo-2H-chromen-4yl)amino)phenyl)morpholine-3-one (YS-07): Yield: $58 \%$; m.p.: $210-212{ }^{\circ} \mathrm{C}$; m.f.: $\mathrm{C}_{21} \mathrm{H}_{19} \mathrm{~N}_{3} \mathrm{O}_{6}$, m.w.: 409.13 , Appearance: Off white, $R_{\mathrm{f}}$ value- 0.40 ( $8: 2$ ethyl acetate:hexane), ${ }^{1} \mathrm{H}$ NMR spectra in $\delta \mathrm{ppm}: 2.42 \delta \mathrm{ppm}$ (singlet, $6 \mathrm{H},-\mathrm{CH}_{3}$ ), 3.78 to 3.89 $\delta \mathrm{ppm}$ (triplet, $2 \mathrm{H},-\mathrm{N}_{-} \mathrm{CH}_{2}-$ ), 4.10 to $4.18 \delta \mathrm{ppm}$ (triplet, $2 \mathrm{H}$, $\left.-\mathrm{CO}-\mathrm{CH}_{2-}\right), 6.89$ to $7.89 \delta \mathrm{ppm}(6 \mathrm{H}$, aromatic region), $11.05 \delta$ ppm (singlet, $1 \mathrm{H},-\mathrm{NH})$. IR ( $\left.\mathrm{KBr}, \nu_{\max }, \mathrm{cm}^{-1}\right)$ : 3780, 3734, 3663, $3642,3391,3268,2920,2370,2354,2280,1669,1657,1597$, $1557,1521,1486,1417,1367,1312,1238,1193,1125,1067$, $997,875,820,766,750,654$. Mass spectra $m / z: 409.13(100.00$ $\%), 410.13(23.2 \%), 411.13(4.0 \%), 410.12(1.1 \%), 395$, $378,365,361,349,332,306,289,275,262,249,223,278$, $205,{ }^{13} \mathrm{C}$ NMR (100 MHz, chloroform- $d$ ) $\delta 125.00,123.30$, 122.67, 121.53, 19.01, 12.44. Elemental analysis (\%): C, 61.61; H, 4.68; N, 10.26; Found (\%): C, 60.02; H, 4.37; N, 9.53. 
4-(4-((6-Methyl-3-nitro-2-oxo-2H-chromen-4-yl)amino)phenyl)morpholine-3-one (YS-08): Yield: $67 \%$; m.p.: 164-166 ${ }^{\circ}$ C; m.f.: $\mathrm{C}_{20} \mathrm{H}_{17} \mathrm{~N}_{3} \mathrm{O}_{6}$, m.w.: 395.37, Appearance: Off white, $R_{\mathrm{f}}$ value- 0.40 (8:2 ethyl acetate:hexane), ${ }^{1} \mathrm{H}$ NMR spectra in $\delta \mathrm{ppm}: 2.44 \delta \mathrm{ppm}$ (singlet, $3 \mathrm{H},-\mathrm{CH}_{3}$ ), 3.80 to $3.84 \delta \mathrm{ppm}$ (triplet, $2 \mathrm{H},-\mathrm{N}_{-} \mathrm{CH}_{2}$ ), 4.05 to $4.08 \delta \mathrm{ppm}$ (triplet, $2 \mathrm{H}$, -CO$\left.\mathrm{CH}_{2}-\right), 6.91$ to $7.75 \delta \mathrm{ppm}(7 \mathrm{H}$, aromatic region), $11.01 \delta \mathrm{ppm}$ (singlet, $1 \mathrm{H},-\mathrm{NH})$. IR (KBr, $\left.\nu_{\max }, \mathrm{cm}^{-1}\right): 3780,3733,3692$, 3398, 3257, 2911, 2391, 2365, 2281, 1690, 1653, 1598, 1553, 1510, 1484, 1416, 1366, 1314, 1238, 1193, 1122, 1050, 999, $875,820,768,751,654$. Mass spectra $m / z: 395.11(100.0 \%)$, $396.12(22.1 \%), 397.12$ (3.6 \%), 396.11 (1.1\%), 378, 365, $349,331,306,289,275,262,249,224,219,206,{ }^{13} \mathrm{C}$ NMR $(100 \mathrm{MHz}$, chloroform- $d$ ) $\delta 133.73,123.97,123.30,121.53$, 115.73, 20.71. Elemental analysis (\%): C, 60.76; H, 4.33; N, 10.63; Found (\%): C, 60.70; H, 4.21; N, 9.40.

4-(4-((7-Methyl-3-nitro-2-oxo-2H-chromen-4-yl)amino)phenyl)morpholine-3-one (YS-09): Yield: 77 \%; m.p.: 186$188^{\circ}$ C; m.f.: $\mathrm{C}_{20} \mathrm{H}_{17} \mathrm{~N}_{3} \mathrm{O}_{6}$, m.w.: 395.37, Appearance: Off white, $\mathrm{R}_{\mathrm{f}}$ value-0.41 (8:2 ethyl acetate:hexane), ${ }^{1} \mathrm{H}$ NMR spectra in $\delta$ ppm: $2.43 \delta \mathrm{ppm}$ (singlet, $3 \mathrm{H},-\mathrm{CH}_{3}$ ), 3.75 to $3.82 \delta \mathrm{ppm}$ (triplet, $2 \mathrm{H},-\mathrm{N}_{-} \mathrm{CH}_{2}$ ), 4.04 to $4.08 \delta \mathrm{ppm}$ (triplet, $2 \mathrm{H}$, -CO$\left.\mathrm{CH}_{2}-\right), 6.92$ to $7.76 \delta \mathrm{ppm}(7 \mathrm{H}$, aromatic region), $11.06 \delta \mathrm{ppm}$ (singlet, $1 \mathrm{H},-\mathrm{NH}), \mathrm{IR}\left(\mathrm{KBr}, v_{\max }, \mathrm{cm}^{-1}\right)$ : $3780,3733,3692$, 3398, 3257, 2911, 2391, 2365, 2281, 1690, 1653, 1598, 1553, 1510, 1484, 1416, 1366, 1314, 1238, 1193, 1122, 1050, 999, $875,820,768,751,654$. Mass spectra $m / z: 395.11(100.0 \%)$, $396.12(22.1 \%), 397.12(3.6 \%), 396.11$ (1.1\%), 378, 365, $361,348,332,306,289,274,262,249,224,219,204 .{ }^{13} \mathrm{CNMR}$ $(100 \mathrm{MHz}$, chloroform- $d$ ) $\delta 126.08,123.69,123.30,121.53$, 115.31, 22.22. Elemental analysis (\%): C, 60.76; H, 4.33; N, 10.63; Found (\%): C, 60.70; H, 4.21; N, 9.40.

4-(4-((8-Methyl-3-nitro-2-oxo-2H-chromen-4-yl)amino)phenyl)morpholine-3-one (YS-10): Yield: $75 \%$; m.p.: 180-182 ${ }^{\circ}$ C; m.f.: $\mathrm{C}_{20} \mathrm{H}_{17} \mathrm{~N}_{3} \mathrm{O}_{6}$, m.w.: 395.37, Appearance: Off white, $\mathrm{R}_{\mathrm{f}}$ value-0.40 (8:2 ethyl acetate:hexane), ${ }^{1} \mathrm{H}$ NMR spectra in $\delta$ ppm: $2.45 \delta \mathrm{ppm}$ (singlet, $3 \mathrm{H},-\mathrm{CH}_{3}$ ), 3.80 to $3.82 \delta \mathrm{ppm}$ (triplet, $2 \mathrm{H},-\mathrm{N}_{-} \mathrm{CH}_{2}$ ), 4.05 to $4.07 \delta \mathrm{ppm}$ (triplet, $2 \mathrm{H}$, -CO$\left.\mathrm{CH}_{2}-\right), 6.90$ to $7.75 \delta \mathrm{ppm}(7 \mathrm{H}$, aromatic region), $11.00 \delta \mathrm{ppm}$ (singlet, $1 \mathrm{H},-\mathrm{NH}-)$, IR (KBr, $\left.v_{\max }, \mathrm{cm}^{-1}\right): 3777,3732,3693$, $3643,3399,3258,2910,2391,2364,2281,1692,1653,1599$, 1553, 1511, 1484, 1416, 1367, 1315, 1238, 1193, 1121, 1058, 999, 875, 820, 767, 751, 655. Mass spectra $\mathrm{m} / \mathrm{z}: 395\left(\mathrm{M}^{+}\right)$, 378, 365, 361, 349, 332, 306, 289, 275, 262, 249, 223, 218, 205. ${ }^{13} \mathrm{C}$ NMR (100 MHz, chloroform- $d$ ) $\delta 133.86,123.30,122.42$, 122.22, 121.53, 15.96, Elemental analysis (\%): C, 60.76; H, 4.33; N, 10.63; Found (\%): C, 60.70; H, 4.21; N, 9.40.

\section{RESULTS AND DISCUSSION}

We have prepared a library of novel 4-(4-amino phenyl)morpholine-3-one containing different coumarin derivatives by chloroamine coupling reaction using inorganic base and DMF as solvent at low temperature which results is 4-(4-((3nitro-2-oxo-2 $H$-chromen-4-yl)amino)phenyl)morpholine-3one derivatives. The formation of 4-(4-((3-nitro-2-oxo- $2 \mathrm{H}$ chromen-4-yl)amino)phenyl)morpholine-3-one by this method was first developed by us. All the synthesized compounds were obtained in good to moderate yield. All synthesized compounds were characterized by IR, NMR and mass spectroscopy.

\section{CONFLICT OF INTEREST}

The authors declare that there is no conflict of interests regarding the publication of this article.

\section{REFERENCES}

1. A. Zalfiqar and H. Nasim, Indian J. Chem., 46B, 1322 (2007).

2. B.S. Creaven, D.A. Egan, K. Kavanagh, M. McCann, A. Noble, B. Thati and M. Walsh, Inorg. Chim. Acta, 359, 3976 (2006); https://doi.org/10.1016/j.ica.2006.04.006

3. P. Laurin, D. Ferroud, M. Klich, C. Dupuis-Hamelin, P. Mauvais, P. Lassaigne, A. Bonnefoy and B. Musicki, Bioorg. Med. Chem. Lett., 9, 2079 (1999);

https://doi.org/10.1016/S0960-894X(99)00329-7.

4. K. Abou-Melha and H. Faruk, J. Iran. Chem. Soc., 5, 122 (2008); https://doi.org/10.1007/BF03245825.

5. M. Di Braccio, G. Grossi, G. Roma, M.G. Signorello and G. Leoncini, Eur. J. Med. Chem., 39, 337 (2004); https://doi.org/10.1016/j.ejmech.2003.12.010.

6. I. Khan, M. Kulkarni and C.-M. Sun, Eur. J. Med. Chem., 40, 1168 (2005);

https://doi.org/10.1016/j.ejmech.2005.05.007.

7. A. Burguete, E. Pontiki, D. Hadjipavlou-Litina, S. Ancizu, R. Villar, B. Solano, E. Moreno, E. Torres, S. Pérez, I. Aldana and A. Monge, Chem. Biol. Drug Des., 77, 255 (2011);

https://doi.org/10.1111/j.1747-0285.2011.01076.x.

8. L.E. Seitz, W.J. Suling and R.C. Reynolds, J. Med. Chem., 45, 5604 (2002);

https://doi.org/10.1021/jm020310n.

9. J. Guillon, I. Forfar, M. Mamani-Matsuda, V. Desplat, M. Saliège, D. Thiolat, S. Massip, A. Tabourier, J.-M. Léger and B. Dufaure, Bioorg. Med. Chem., 15, 194 (2007);

https://doi.org/10.1016/j.bmc.2006.09.068.

10. V.K. Tandon, B.D. Yadav, H.K. Maurya, A.K. Chaturvedi and P.K. Shukla, Bioorg. Med. Chem., 14, 6120 (2006); https://doi.org/10.1016/j.bmc.2006.04.029.

11. B. Zarranz, A. Jaso, I. Aldana and A. Monge, Bioorg. Med. Chem., 12, 3711 (2004); https://doi.org/10.1016/j.bmc.2004.04.013.

12. M. Waring, T. Ben-Hadda, A. Kotchevar, A. Ramdani, R. Touzani, S. Elkadiri, A. Hakkou, M. Bouakka and T. Ellis, Molecules, 7, 641 (2002); https://doi.org/10.3390/70800641.

13. E. Vicente, L.M. Lima, E. Bongard, S. Charnaud, R. Villar, B. Solano, A. Burguete, S. Perez-Silanes, I. Aldana and L. Vivas, Eur. J. Med. Chem., 43, 1903 (2008); https://doi.org/10.1016/j.ejmech.2007.11.024.

14. Y.B. Kim, Y.H. Kim, J.Y. Park and S.K. Kim, Bioorg. Med. Chem., 14, 541 (2004); https://doi.org/10.1016/j.bmcl.2003.09.086.

15. J. Jampilek, Curr. Med. Chem., 21, 4347 (2014); https://doi.org/10.2174/0929867321666141011194825.

16. K. Toshima, K. Takano, T. Ozawa and S. Matsumura, Chem. Commun., 212 (2002); https://doi.org/10.1039/b107829c.

17. N.D. Sonawane and D. Rangnekar, Heterocycl. Chem., 39, 303 (2002); https://doi.org/10.1002/jhet.5570390210.

18. A. Katoh, T. Yoshida and J. Ohkanda, Heterocycles, 52, 911 (2000); https://doi.org/10.3987/COM-99-S61.

19. J.F. Zhou, G.X. Gong, L.T. An, Y. Liu, Y.X. Zhu, Y.L. Zhu and S.-J. Ji, Synlett, 2008, 3163 (2008); https://doi.org/10.1055/s-0028-1087280.

20. A.A. Kamble, R.R. Kamble, M.N. Kumbar and G. Tegginamath, Med. Chem. Res., 25, 1163 (2016); https://doi.org/10.1007/s00044-016-1558-2. 